\title{
Comparison of the effects of transcutaneous electrical nerve stimulation, low level laser, and placebo treatment on temporomandibular joint disorders: a single-blind randomized controlled trial
}

\author{
Hyunjoong Kim ${ }^{\mathrm{a}, \mathrm{b}}$ (1) \\ ${ }^{a}$ Sports Rehabilitation Center, The Better Hospital, Gwangju, Republic of Korea \\ ${ }^{b}$ Department of Physical Therapy, Gwangju Health University, Gwangju, Republic of Korea
}

Objective: Physical therapy techniques are required for patients with temporomandibular joint disorder (TMD), but the effects of treatment have not been compared. Therefore, effects of transcutaneous electrical nerve stimulation (TENS) and low level laser (LLL), which are most commonly used interventions, were compared.

Design: Randomized controlled trial.

Methods: Thirty-six participants with pain in the temporomandibular joint were enrolled, and 12 participants were randomly assigned to either the TENS group, LLL group, or placebo group. Each intervention was performed for a total of 6 sessions for 2 weeks. For the evaluation of the participants, the mouth opening (MO), pressure pain threshold (PPT), and stress were measured at three time periods: baseline, post-test, and follow-up at 2 weeks.

Results: Significant interaction between groups according to each evaluation point was found only in PPT-masseter $(p<0.05)$. The evaluation time point at which a significant difference appeared was at the post-test and follow-up at 2 weeks time periods. As a result of the post-test, the LLL group showed a significant improvement compared to the TENS group $(p<0.05)$, and at 2 weeks follow-up, the TENS group showed a significant improvement compared to the placebo group $(p<0.05)$.

Conclusions: In this study, an experiment was conducted to compare the treatment effects when TENS, LLL, and placebo were given to patients with TMD. In addition, by quantitatively presenting the effect size of each treatment, this study suggests clinical use of TENS and LLL treatment for TMD.

Key Words: Pain threshold, Psychological stress, Range of motion, Temporomandibular joint disorders

\section{Introduction}

The temporal mandibular joint (TMJ) is a bi-arthroidal hinge joint that allows daily complex movements in relation to mastication activities [1]. Cases involving disorders of the TMJ and related anatomical structures are defined as temporomandibular joint disorder (TMD) [2]. TMD occurs in a large number of people worldwide, with about $25 \%$ of adults presenting signs or symptoms [3]. In addition, it affects female at a rate of 1.5 to 2.5 times higher than male [4].
The etiology of TMD is multidimensional given that neurobiological, biomechanical, neuromuscular, and biopsychosocial factors can contribute to the disability [5]. In general, the cause of the pain caused by TMD is the disc displacement, and the disc displacement without reduction often has pain and mouth opening limitation $[6,7]$. In particular, pain with TMD is a decisive outcome even though it is not a pathologically direct cause, and affects the patient's function and quality of life [8].

There are various physical therapy techniques for treat-

Received: 4 December, 2020 Revised: 29 December, 2020 Accepted: 29 December, 2020

Corresponding author: Hyunjoong Kim (ORCID https://orcid.org/0000-0001-6538-3872)

Department of Physical Therapy, Gwangju Health University, 73 Bungmun-daero 419beon-gil, Gwangsan-gu, Gwangju 62287, Republic of Korea Tel: 82-62-958-7786 Fax: 82-62-958-7786 E-mail: doong18324@gmail.com

(c) This is an Open-Access article distributed under the terms of the Creative Commons Attribution Non-Commercial License (http://creativecommons.org/licenses/ by-nc/4.0) which permits unrestricted non-commercial use, distribution, and reproduction in any medium, provided the original work is properly cited.

Copyright $@ 2020$ Korean Academy of Physical Therapy Rehabilitation Science 
ment of TMD. Treatment methods include psychotherapy, acupuncture, transcutaneous electrical nerve stimulation (TENS), soft tissue mobilization, low level laser (LLL), TMJ mobilization, and exercise therapy [9-13].

Among these various physical therapy techniques, TENS is well known as an effective and safe non-invasive method for pain control. TENS can reduce pain through increased blood flow by causing regular contraction of muscles, and is effective in pain control based on the gate control theory [14]. In addition, LLL, which is commonly used clinically for pain control for TMD, is widely used in musculoskeletal diseases. The mechanism of pain control of LLL has not been clearly identified, but it is known to decrease the permeability of nerve cell membranes by reducing edema due to an increase in microcirculation $[15,16]$.

Many studies have been conducted on the treatment for TMD, which accounts for a large proportion of musculoskeletal disorders, but suggesting an effective method is limited. Therefore, the purpose of this study was to compare the therapeutic effects of TENS and LLL, which are mainly used in clinical practice, and apply a placebo to investigate each treatment effect.

\section{Methods}

\section{Participants}

This study was recruited from adults who complained of TMD symptoms on the bulletin board of Paul Block at Gwangju Health University. The inclusion criteria for participants was based on a simplified questionnaire for TMD of the American Academy of Orofacial Pain [17]. A simple questionnaire can be judged as TMD if at least one of the 10 items is positive. One symptom is considered as mild, two to 3 symptoms are considered as moderate, and four or more are considered to be severe. The exclusion criteria was patients with a history of recent trauma or other diseases in the area, people with cardiac arrhythmias or pacemakers, and those who were pregnant [18]. In this study, among potential participants, if pain occurred during left and right TMJ palpation when opening the mouth, the details and purpose of the study were informed, and if they agreed to participate, they were enrolled in the study. A total of 36 participants enrolled in this study where 12 participants were assigned to one of three groups according to a randomization method.

This study was approved by the Institutional Review Board of Sahmyook University (2-1040781-AB-N-01-2017 $050 \mathrm{HR}$ ) before the start of the experiment, and the random- ized controlled trial was enrolled in the Clinical Research Information Service (KCT0002834).

The sample size was estimated as the amount of change in maximal mouth opening through LLL [18]. The calculated effect size $f(V)$ was 0.37 , and the power was 0.95 using G*Power 3.1 (Universität Kiel, Kiel, Germany), and when 3 groups and 3 measurements were taken, 27 participants were calculated. In consideration of dropouts $(30 \%)$ during the experiment, 36 participants were finally recruited.

\section{Randomization and blinding}

The participants were randomly assigned to TENS, LLL, or placebo groups using a Random Allocation Software (Isfahan University, Isfahan, Iran). Randomization was randomly generated with 3 blocks and 4 digits. The blindfolding of participants prevented them from knowing what treatment they were receiving by different intervention schedules.

\section{Intervention}

In order to minimize errors that may occur in the procedure during the experiment, education on the research procedure was conducted before the intervention and evaluation. Each intervention was conducted over 2 weeks, 3 times a week, 10 minutes per session, for a total of 6 sessions.

\section{Transcutaneous electrical nerve stimulation}

For TENS, Intelect Advanced Color Combo with EMG (Chattanooga, Chattanooga, TN, USA) was used. The pad size was $20 \times 20 \mathrm{~mm}$, the waveform was symmetrical biphasic, the phase duration was $50 \mu \mathrm{s}$, and the frequency was 4 Hz. Intensity was applied by adjusting the amplitude until the participant's muscle contraction occurred and pain was felt. The electrode location was a dual channel arrangement method widely used in the participant's pain area. In addition, the active electrode and the inactive electrode of each pad were attached to a place in contact with the sternocleidomastoid (SCM) muscle and the masseter muscle in a unilateral arrangement [19].

\section{Low level laser}

LLL is a non-invasive method for optical stimulation. The Laserneedle Touch (Laserneedle GmbH, Glienicke/Nordbahn, Germany) used in this study provided a continuous wave mode red light and $685 \mathrm{Nm}$ that generates about $2.3 \mathrm{~kJ} / \mathrm{cm}^{2}$ of radiation exposure energy for 10 minutes of stimulation time [20,21]. It emits an output or frequency modulated laser 
light of $40 \mathrm{~mW}$ per laser in wavelength. The treatment site was the masseter and the SCM. The masseter is the area connected widely between the zygomatic arch and the mandible, and the SCM spans from the sternum and clavicle to the mastoid process of the temporal bone [19].

\section{Placebo}

The placebo was performed by attaching a laser pad after blocking the participant's vision in the same way as other interventions.

\section{Outcome measure}

The mouse opening (MO), pressure pain threshold (PPT), and stress were evaluated three times in total (baseline, post-test, and follow-up at 2 weeks).

\section{Mouth opening}

In this study, TMJ maximal mouth opening was measured using the Therabite Range of Motion Scale (TRMS). TRMS was created for self-diagnosis of TMD patients, and is a 'gold-standard' grade measurement tool with high reliability of patient measurement reliability $r=0.92$ [22]. The median value was recorded by repeatedly measuring the maximum opening within the range of the participant's active pain-free position in a comfortable lying position with a $\mathrm{mm}$ ruler at the interval between the median rows of the upper and lower teeth. The range of normal mouth opening is 40-50 mm.

\section{Pressure pain threshold}

In this study, the PPT was measured using a pressure pain gauge. As a pain measurement tool, each of the pain thresholds was measured at the painful site of the TMJ using a Commander Algometer (JTECH Medical Industries, Inc., Salt Lake City, UT, USA). The measuring instrument was placed vertically on the SCM and masseter muscles. The pressure was increased at a constant rate of $1 \mathrm{lb} / \mathrm{sec}$, and made a "ah" beep when the pain began to be felt, and the pressure applied at that moment was measured as the tenderness threshold. To reduce the error, evaluation was performed 3 times at 1-minute intervals and average values were recorded. The intra-measure reliability is $\mathrm{r}=0.99$ [23].

\section{Stress}

Stress is an emotional factor that can cause tension and illness. Among them, as a physiological reaction, the function of the cardiovascular system is enhanced, and sweating occurs. The secretory activity of the sweat glands can be measured by skin conductance, and the cardiovascular system is measured by heart rate. Stress measurement was performed using ProComp Infiniti (Thought Technology, Montreal, QC, Canada) [24]. The unit of skin conduction is micro suemens ( $\mu \mathrm{s}$ ), the normal range is $2 \mu \mathrm{s}$ or less, the unit of heart rate is beat $/ \mathrm{min}$, and the normal range is $60-80$ beat $/ \mathrm{min}$. As a measurement method, a skin conduction sensor was attached to the right index finger and little finger for skin conduction, and a heart rate sensor was attached to the right middle finger for heart rate.

\section{Statistical analysis}

All statistical analysis was performed using IBM SPSS for Windows, Version 25.0 (IBM Co., Armonk, NY, USA). The homogeneity test used a chi-square test for categorical variables and one-way analysis of variance (ANOVA) for continuous variables. The main results were measured using a two-way repeated measures ANOVA to confirm the interaction between time and group. When there were significant differences, differences between groups were analyzed using a one-way ANOVA and Bonfferroni's post-hoc test. In addition, partial eta square $\left(\eta_{\mathrm{p}}^{2}\right)$ and Cohen's d (Cohen's criteria: small $\leq 0.2$; moderate $=0.5$; large $\geq 0.8$ ) were used to determine the effect size of treatment according to each intervention. All statistical significance levels were set to 0.05. In addition, in the post-hoc test, the Bonfferoni test was divided by the number of groups comparing the significance level of 0.05 . Therefore, since it was 3 groups, the significance level was set to 0.017 .

\section{Results}

All 36 participants completed the study without withdrawing from the study (Figure 1). The general characteristics of participants are shown in Table 1, and there was no significant difference between groups $(p>0.05)$.

\section{Mouth opening}

As a result of $\mathrm{MO}$, there were no statistically significant interactions according to the evaluation time point of each group ( $\left.p>0.05, \eta_{\mathrm{p}}^{2}=0.107\right)$. However, in the results of comparison through effect size, the post-intervention effect of the LLL group was the greatest $(\mathrm{d}=3.07)$. The differences in MO are summarized in Tables 2, 3 . 


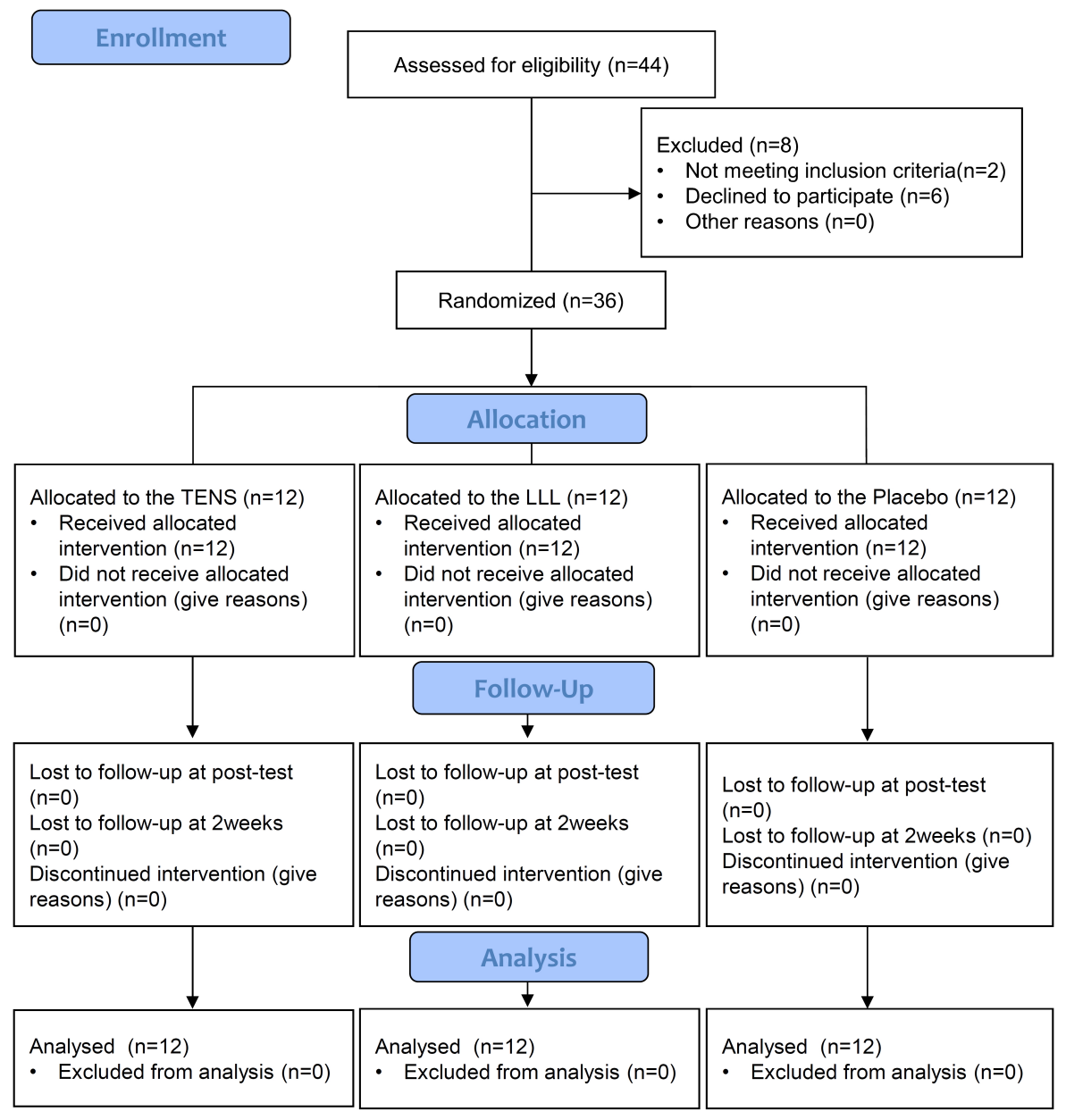

Figure 1. Flow diagram of participant recruitment, allocation, and analysis. TENS: transcutaneous electrical nerve stimulation, LLL: low level laser.

Table 1. General characteristics of participants

\begin{tabular}{lcccc}
\hline & TENSG $(\mathrm{n}=12)$ & LLLG $(\mathrm{n}=12)$ & PG $(\mathrm{n}=12)$ & $\chi^{2} / \mathrm{F}$ \\
\hline Age $(\mathrm{y})$ & $23.50(2.07)$ & $21.75(2.86)$ & $21.58(3.20)$ & 1.784 \\
Sex $($ male/female $)$ & $7 / 5$ & $7 / 5$ & $5 / 7$ & 0.892 \\
Height $(\mathrm{cm})$ & $171.58(7.22)$ & $169.67(10.11)$ & $168.33(11.74)$ & 0.329 \\
Weight $(\mathrm{kg})$ & $61.42(9.03)$ & $66.00(14.62)$ & $60.33(11.48)$ & 0.762 \\
BMI $\left(\mathrm{kg} / \mathrm{m}^{2}\right)$ & $20.76(1.80)$ & $22.64(2.79)$ & $21.08(1.34)$ & 2.847 \\
\hline
\end{tabular}

Values are presented as number only or mean (SD).

BMI: body mass index, TENSG: transcutaneous electrical nerve stimulation group, LLLG: low level laser group, PG: placebo group.

\section{Pressure pain threshold}

As a result of PPT-masseter, there was a statistically significant interaction according to the evaluation time point of each group $\left(p<0.05, \eta_{\mathrm{p}}{ }_{\mathrm{p}}=0.219\right)$. Significant differences at each evaluation point were at the post-test and 2-week follow-up time periods. Post-test results showed significant improvement in the LLL group compared to TENS group $(p<0.017)$, and at 2 weeks follow-up, significant improve- ments were seen in the TENS group compared to the placebo group $(p<0.017)$. The differences in PPT-masseter are summarized in Tables 2, 3 .

As a result of PPT-SCM, there was no statistically significant interactions according to the evaluation time point of each group $\left(p>0.05, \eta_{\mathrm{p}}^{2}=0.108\right)$. However, in the result of comparing the effect size, the carryover effect of the TENS group was the greatest $(\mathrm{d}=2.31)$. The differences in PPT-SCM are summarized in Tables 2, 3. 
Table 2. Results for each group according to the evaluation time point

\begin{tabular}{|c|c|c|c|c|c|}
\hline & TENSG $(n=12)$ & LLLG $(\mathrm{n}=12)$ & PG $(n=12)$ & Time $\times$ Group & $\eta_{\mathrm{p}}^{2}$ \\
\hline \multicolumn{6}{|l|}{ Mouse opening (mm) } \\
\hline Baseline & $30.83(4.37)$ & $33.61(3.81)$ & $32.58(2.69)$ & 1.972 & 0.107 \\
\hline Post-test & $37.17(7.87)$ & $43.28(2.30)$ & $38.69(4.09)$ & & \\
\hline Follow-up at 2 weeks & $37.06(7.28)$ & $39.72(4.26)$ & $34.95(6.19)$ & & \\
\hline \multicolumn{6}{|l|}{$\begin{array}{l}\text { Pressure pain threshold; } \\
\text { masseter (lb) }\end{array}$} \\
\hline Baseline & $3.49(0.38)$ & $3.78(0.80)$ & $3.74(0.72)$ & $4.627^{*}$ & 0.219 \\
\hline Post-test & $3.70(0.35)$ & $4.67(0.75)^{\mathrm{a}}$ & $3.57(0.61)$ & & \\
\hline Follow-up at 2 weeks & $4.53(0.84)^{b}$ & $5.58(1.24)$ & $4.03(0.75)$ & & \\
\hline \multicolumn{6}{|c|}{$\begin{array}{l}\text { Pressure pain threshold; } \\
\text { sternocleidomastoid (lb) }\end{array}$} \\
\hline Baseline & $3.77(0.55)$ & $4.51(1.47)$ & $4.29(1.91)$ & 2.005 & 0.108 \\
\hline Post-test & $4.33(0.73)$ & $5.10(0.60)$ & $3.89(1.13)$ & & \\
\hline Follow-up at 2 weeks & $4.95(0.47)$ & $5.39(0.58)$ & $4.23(1.21)$ & & \\
\hline \multicolumn{6}{|c|}{ Stress; skin conductance $(\mu \mathrm{s})$} \\
\hline Baseline & $4.60(2.09)$ & $3.85(4.04)$ & $3.22(2.47)$ & 6.532 & 0.119 \\
\hline Post-test & $3.32(1.83)$ & $3.45(2.59)$ & $2.53(1.93)$ & & \\
\hline Follow-up at 2 weeks & $3.15(2.10)$ & $4.88(4.75)$ & $2.66(1.76)$ & & \\
\hline \multicolumn{6}{|c|}{ Stress; heart rate (beat $/ \mathrm{min})$} \\
\hline Baseline & $81.14(11.00)$ & $93.94(11.84)$ & 77.45 (20.99) & 0.390 & 0.023 \\
\hline Post-test & $74.95(11.73)$ & $87.88(12.32)$ & $74.43(20.25)$ & & \\
\hline Follow-up at 2 weeks & $84.83(27.69)$ & $92.19(20.70)$ & 86.67 (18.77) & & \\
\hline
\end{tabular}

Values are presented as mean (SD).

TENSG: transcutaneous electrical nerve stimulation group, LLLG: low level laser group, PG: placebo group.

${ }^{\mathrm{a}} p<0.017$ : TENSG $<$ LLLG, ${ }^{\mathrm{b}} p<0.017$ : TENSG $>$ PG.

$*_{p}<0.05$.

Table 3. Comparisons of the effect size for each intervention method using Cohen's d

$(\mathrm{N}=36)$

\begin{tabular}{|c|c|c|c|c|c|}
\hline Variables & Mouse opening & PPT-masseter & PPT-SCM & Stress-SC & Stress-HR \\
\hline \multicolumn{6}{|l|}{ TENSG } \\
\hline${ }^{\mathrm{a}} \mathrm{Pe}$ & 1.00 & 0.57 & 0.86 & 0.65 & 0.54 \\
\hline${ }^{\mathrm{b}} \mathrm{Ce}$ & 1.04 & 1.58 & 2.31 & 0.69 & 0.18 \\
\hline \multicolumn{6}{|l|}{ LLLG } \\
\hline $\mathrm{Pe}$ & 3.07 & 1.14 & 0.53 & 0.12 & 0.50 \\
\hline $\mathrm{Ce}$ & 1.51 & 1.71 & 0.79 & 0.23 & 0.10 \\
\hline \multicolumn{6}{|l|}{ PG } \\
\hline $\mathrm{Pe}$ & 1.76 & 0.26 & 0.25 & 0.31 & 0.15 \\
\hline $\mathrm{Ce}$ & 0.50 & 0.39 & 0.04 & 0.26 & 0.46 \\
\hline
\end{tabular}

Values are presented as effect size.

TENSG: transcutaneous electrical nerve stimulation group, LLLG: low level laser group, PG: placebo group, PPT: pressure pain threshold, PPT-SCM: pressure pain threshold-sternocleidomastoid, SC: stress-skin conductance, HR: heart rate.

${ }^{\text {aPe: }}$ post-intervention effect: post-test minus baseline.

${ }^{\mathrm{b}} \mathrm{Ce}$ : carryover effect: follow-up at 2 weeks minus baseline.

\section{Stress}

As a result of stress-skin conductance, there were no statistically significant interactions according to the evaluation time point of each group $\left(p>0.05, \eta_{\mathrm{p}}^{2}=0.119\right)$. However, in the result of comparing the effect size, the carryover effect of the TENS group was the greatest $(d=0.69)$. The differences in stress-skin conductance are summarized in Tables 2, 3.

As a result of the stress-heart rate, there were no statistically significant interactions according to the evaluation 
time point of each group $\left(p>0.05, \eta_{\mathrm{p}}^{2}=0.023\right)$. However, in the result of comparing the effect size, the post-intervention effect of the TENS group was the greatest $(\mathrm{d}=0.54)$. The differences in stress-heart rate are summarized in Tables 2, 3.

\section{Discussion}

TMD, which accounts for a large part of musculoskeletal pain, affects quality of life due to pain and limitation of chewing activity. Although studies on various treatment methods have been conducted, studies on the therapeutic effects of TENS and LLL, which are easy to apply and effective methods in clinical practice, are insufficient. Therefore, in this study, the effects of each treatment were compared and the effect of each treatment method was quantitatively analyzed through the application of a placebo.

As a result of this study, significant interaction between groups according to each evaluation time point was found in PPT-masseter $(p<0.05)$. The evaluation time point at which a significant difference appeared was post-test and follow-up at 2 weeks. In the post-test, the LLL group showed a significant improvement over the TENS group $(p<0.017)$, and at 2 weeks follow-up, the TENS group showed a significant improvement over the placebo group $(p<0.017)$. In addition, there were no significant interactions with other variables, but the treatment effect was quantified by the effect size (Cohen's d) for each treatment method. In the results of MO through effect size, a large effect (large $\geq 0.8$ ) was found in the post-intervention effect $(d=3.07)$ and the carryover effect $(\mathrm{d}=1.51)$ in the LLL group. In addition, remarkable large effects were observed in the post-intervention effects $(d=0.86)$ and carryover effects $(d=2.31)$ in the TENS group in PPT-SCM.

In the MO results of this study, there was no significant difference between groups according to each evaluation point. As in this study, studies comparing TENS and LLL for TMJ also showed no significant difference [18,25]. Conversely, there was a study that showed a significant improvement in MO when applying LLL [19]. However, this is also a result that is consistent with the results analyzed through the effect size in this study. In addition, other studies used general LLL, and in this study, acupuncture-type LLL was used. This was similar to the average difference of 8 $\mathrm{mm}$, similar to other previous studies [26].

In the PPT-masseter results of this study, there were significant differences between groups according to each evaluation time point $(p<0.05)$. In the post-test period, the LLL group showed a significant improvement over the TENS group $(p<0.017)$, and at 2 weeks follow-up, the TENS group showed a significant improvement over the placebo group $(p<0.017)$. However, there was no significant interaction in the results of PPT-SCM, and there was a large effect in the TENS group in comparison through effect size. In other previous studies similar to this study, there was a significant effect on the pain control of the masseter through laser therapy [25], and significantly improved muscle activity when using acupuncture-type laser [27]. As a result of the previous studies, it is thought that LLL was more effective in pain control than TENS, and it was more significant due to the increased masseter muscle activity and decreased myofascial pain.

In the stress results of this study, there was no significant difference between groups according to each evaluation point. In comparison through effect size, skin conductance had a post-intervention effect and a carryover effect as a moderate effect in the TENS group, and a post-intervention effect as a moderate effect in the TENS group and LLL group for heart rate. In the study of Monaco et al. [28], which partially coincided with the results of this study, the result of comparing stress indices under stress conditions using ultra low frequency transcutaneous electric nervous stimulation [29], which are effective for TMD treatment, showed that compared to the TENS group, the control group showed a higher increase in stress, therefore, TENS was effective in reducing stress.

As a limitation of this study, recruitment of participants was limited to universities and the sample size was small. In addition, the quality of life due to pain and dysfunction was attempted to be confirmed through stress, but precise analysis was difficult, and it is believed that TENS and LLL had somewhat different effects on the participant. This is because in the case of laser, the difference between the treatment duration and irradiated area may change, and in the case of TENS, the current delivered to the participant may cause great discomfort to some patients.

Therefore, in further studies, it is expected that discussion on the quality of life by recruiting a large number of participants in actual clinical practice is necessary, and it is necessary to restate each treatment effect by configuring the placebo in more detail.

It was conducted under the assumption that TMD-induced pain (PPT) causes dysfunction (MO) and affects stress (skin conductance and heart rate), and the effects of each treatment method (TENS, LLL, and placebo) were also compared. 
LLL was most effective in pain control caused by TMD, and in the long term, TENS was also effective in pain control. In addition, the treatment effect of LLL was higher in the post-intervention effect compared to the placebo, and therefore, it can be said that it is effective for immediate functional improvement.

\section{Conflict of Interest}

The authors declared no potential conflicts of interest with respect to the research, authorship, and/or publication of this article.

\section{References}

1. Di Fabio RP. Physical therapy for patients with TMD: a descriptive study of treatment, disability, and health status. J Orofac Pain 1998;12:124-35.

2. Shaffer SM, Brismée JM, Sizer PS, Courtney CA. Temporomandibular disorders. Part 1: anatomy and examination/diagnosis. J Man Manip Ther 2014;22:2-12.

3. Perez CV, de Leeuw R, Okeson JP, Carlson CR, Li HF, Bush HM, et al. The incidence and prevalence of temporomandibular disorders and posterior open bite in patients receiving mandibular advancement device therapy for obstructive sleep apnea. Sleep Breath 2013;17:323-32.

4. Machado LP, Nery Cde G, Leles CR, Nery MB, Okeson JP. The prevalence of clinical diagnostic groups in patients with temporomandibular disorders. Cranio 2009;27:194-9.

5. Martins WR, Blasczyk JC, Aparecida Furlan de Oliveira M, Lagôa Gonçalves KF, Bonini-Rocha AC, Dugailly PM, et al. Efficacy of musculoskeletal manual approach in the treatment of temporomandibular joint disorder: a systematic review with meta-analysis. Man Ther 2016;21:10-7.

6. Peck CC, Goulet JP, Lobbezoo F, Schiffman EL, Alstergren P, Anderson GC, et al. Expanding the taxonomy of the diagnostic criteria for temporomandibular disorders. J Oral Rehabil 2014; 41:2-23.

7. Pesquera-Velasco J, Casares-García G, Jiménez-Pasamontes N, García-Gómez FA. Method of help for the diagnosis of the temporomandibular joint internal derangements. Discriminant analysis applied to the temporomandibular derangements. Med Oral Patol Oral Cir Bucal 2005;10:294-300.

8. Tjakkes GH, Reinders JJ, Tenvergert EM, Stegenga B. TMD pain: the effect on health related quality of life and the influence of pain duration. Health Qual Life Outcomes 2010;8:46.

9. Roldán-Barraza C, Janko S, Villanueva J, Araya I, Lauer HC. A systematic review and meta-analysis of usual treatment versus psychosocial interventions in the treatment of myofascial temporomandibular disorder pain. J Oral Facial Pain Headache 2014;28:205-22.

10. Fricton J. Current evidence providing clarity in management of temporomandibular disorders: summary of a systematic review of randomized clinical trials for intra-oral appliances and oc- clusal therapies. J Evid Based Dent Pract 2006;6:48-52.

11. Butts R, Dunning J, Pavkovich R, Mettille J, Mourad F. Conservative management of temporomandibular dysfunction: a literature review with implications for clinical practice guidelines (Narrative review part 2). J Bodyw Mov Ther 2017;21:541-8.

12. Gomes CA, Politti F, Andrade DV, de Sousa DF, Herpich CM, Dibai-Filho AV, et al. Effects of massage therapy and occlusal splint therapy on mandibular range of motion in individuals with temporomandibular disorder: a randomized clinical trial. J Manipulative Physiol Ther 2014;37:164-9.

13. Garrigós-Pedrón M, La Touche R, Navarro-Desentre P, GraciaNaya M, Segura-Ortí E. Effects of a physical therapy protocol in patients with chronic migraine and temporomandibular disorders: a randomized, single-blinded, clinical trial. J Oral Facial Pain Headache 2018;32:137-50.

14. Grossmann E, Tambara JS, Grossmann TK, de Siqueira JTT. Transcutaneous electrical nerve stimulation for temporomandibular joint disorder. Rev Dor 2012;13:271-6.

15. Carvalho CM, de Lacerda JA, dos Santos Neto FP, Cangussu MC, Marques AM, Pinheiro AL. Wavelength effect in temporomandibular joint pain: a clinical experience. Lasers Med Sci 2010;25:229-32.

16. Mazzetto MO, Hotta TH, Pizzo RC. Measurements of jaw movements and TMJ pain intensity in patients treated with GaAlAs laser. Braz Dent J 2010;21:356-60.

17. Reiter S, Goldsmith C, Emodi-Perlman A, Friedman-Rubin P, Winocur E. Masticatory muscle disorders diagnostic criteria: the American Academy of Orofacial Pain versus the research diagnostic criteria/temporomandibular disorders (RDC/TMD). J Oral Rehabil 2012;39:941-7.

18. Seifi M, Ebadifar A, Kabiri S, Badiee MR, Abdolazimi Z, Amdjadi P. Comparative effectiveness of low level laser therapy and transcutaneous electric nerve stimulation on temporomandibular joint disorders. J Lasers Med Sci 2017;8(Suppl 1):S2731.

19. Núñez SC, Garcez AS, Suzuki SS, Ribeiro MS. Management of mouth opening in patients with temporomandibular disorders through low-level laser therapy and transcutaneous electrical neural stimulation. Photomed Laser Surg 2006;24:45-9.

20. Litscher G, Wang L, Schwarz G, Schikora D. [Laserneedle stimulation in healthy volonteers and for tinnitus. First computerbased investigations using very early auditory-evoked potentials]. Schweiz Z Ganzheitsmed 2006;18:29-34. German.

21. Litscher G, Schikora D. Cerebral vascular effects of non-invasive laserneedles measured by transorbital and transtemporal Doppler sonography. Lasers Med Sci 2002;17:289-95.

22. Saund DS, Pearson D, Dietrich T. Reliability and validity of self-assessment of mouth opening: a validation study. BMC Oral Health 2012;12:48.

23. Kinser AM, Sands WA, Stone MH. Reliability and validity of a pressure algometer. J Strength Cond Res 2009;23:312-4.

24. Liu Q, Wang Y, van Heck CH, Qiao W. Stress reactivity and emotion in premenstrual syndrome. Neuropsychiatr Dis Treat 2017; 13:1597-602.

25. Kato MT, Kogawa EM, Santos CN, Conti PC. TENS and lowlevel laser therapy in the management of temporomandibular disorders. J Appl Oral Sci 2006;14:130-5.

26. Hu WL, Chang CH, Hung YC, Tseng YJ, Hung IL, Hsu SF. Laser 
acupuncture therapy in patients with treatment-resistant temporomandibular disorders. PLoS One 2014;9:e110528.

27. Hotta PT, Hotta TH, Bataglion C, Bataglion SA, de Souza Coronatto EA, Siéssere S, et al. Emg analysis after laser acupuncture in patients with temporomandibular dysfunction (TMD). Implications for practice. Complement Ther Clin Pract 2010;16:158-60.
28. Monaco A, Cattaneo R, Ortu E, Constantinescu MV, Pietropaoli D. Sensory trigeminal ULF-TENS stimulation reduces HRV response to experimentally induced arithmetic stress: a randomized clinical trial. Physiol Behav 2017;173:209-15.

29. Chipaila N, Sgolastra F, Spadaro A, Pietropaoli D, Masci C, Cattaneo R, et al. The effects of ULF-TENS stimulation on gnathology: the state of the art. Cranio 2014;32:118-30. 